\section{Compressibilities of Cubic Crystalline Powders : a New Method of Measurement}

By suitable averaging, the following relations may be derived for polycrystalline aggregates consisting of randomly oriented and ideally closely packed particles of any substance crystallizing in the cubic system :

$$
\begin{aligned}
& C^{\prime}{ }_{11}=\frac{3}{5} C_{11}+\frac{2}{5}\left(C_{12}+2 C_{44}\right) ; \\
& C_{44}^{\prime}=\frac{3}{5} C_{41}+\frac{1}{5}\left(C_{11}-C_{12}\right) .
\end{aligned}
$$

$C^{\prime}{ }_{11}$ and $C^{\prime}{ }_{44}$ are the thickness and shear elastic constants of a plate of the aggregate and $C_{11}, C_{12}$ and $C_{44}$ are the elastic constants of the corresponding single crystal. If the crystal is such that the Cauchy relation $C_{12}=C_{44}$ holds good, the above equations become dependent on one another and reduce to $C^{\prime}{ }_{11}=3 C_{44}^{\prime}=$ $\frac{3}{5}\left(C_{11}+2 C_{12}\right)$. If $C_{12} \neq C_{44}$, they remain independent but may be combined to give $3 C^{\prime}{ }_{11}-4 C^{\prime}{ }_{44}=C_{11}+$ $2 C_{12}$. Thus, the measurement of $C^{\prime}{ }_{11}$ and $C^{\prime}{ }_{44}$ will enable us not only to state whether the Cauchy relation holds good for the crystal under investigation, but also to evaluate the bulk modulus $K$, which is $\frac{1}{3}\left(C_{11}+2 C_{12}\right)$.

$C^{\prime}{ }_{11}$ and $C^{\prime}{ }_{44}$ can be conveniently measured by the ultrasonic wedge method, developed and described by us in earlier publications ${ }^{1}$. Results obtained for a few test cases, using cubic crystal powders, compressed into plates of suitable dimensions, are given in the

\begin{tabular}{|c|c|c|c|c|}
\hline Substance & $C_{11}^{\prime}$ & $C^{\prime}{ }^{\prime}$, & $K=\frac{5}{9} C_{11}^{\prime}$ & $\underset{\substack{K \\
\text { (direct }}}{ }$ \\
\hline $\begin{array}{l}\mathrm{KCl} \\
\mathrm{K} B r \\
\mathrm{KI}\end{array}$ & $\begin{array}{l}3 \cdot 16 \\
2 \cdot 71 \\
2 \cdot 19\end{array}$ & $\begin{array}{l}1.07 \\
0.91 \\
0 \cdot 76\end{array}$ & $\begin{array}{r}1 \cdot 76 \\
1 \cdot 51 \\
1 \cdot 22\end{array}$ & $\begin{array}{l}1 \cdot 77 \\
1.50 \\
1 \cdot 17\end{array}$ \\
\hline $\mathrm{NaCl}$ & $4 \cdot 37$ & $1 \cdot 47$ & $2 \cdot 43$ & $2 \cdot 40$ \\
\hline
\end{tabular}
accompanying table in units of $10^{11}$ dynes per $\mathrm{cm} .^{2}$.

$C^{\prime}{ }_{11}$ in all cases is three times $C^{\prime}{ }_{44}$. This is in conformity with the known fact that the Cauchy relation holds in all these crystals. Moreover, $K$ obtained from $C^{\prime}{ }_{11}$ (column 4) agrees well with the value available in the literature for each case (column 5).

It is proposed to extend this method to as many aggregates as possible, and the results will be published elsewhere.

Physical Laboratories,

\section{S. Bhagavantam}

T. Seshagiri RaO

Osmania University, Hyderabad 7. May 28. ${ }^{1}$ Bhagavantam, S., and Bhimasenachar, J., Proc. Ind. Acad. Sci.,
20, $298(1944) ;$ Nature, 158, 23 (1945),

\section{Reaction between Glucose and the Terminal Amino Group of Lysine}

Patton and Chism ${ }^{1}$ have recently used paper chromatography followed by examination of the unheated papers under an ultra-violet lamp for demonstrating a series of fluorescent products in boiled glucose-glycinè solutions. We have been using a similar technique in an investigation of the reaction between glucose and $\alpha-\mathrm{N}$-acetyl-L-lysine at $37^{\circ} \mathrm{C}$. and have found it particularly effective when combined with a study of the effect of water content on the course of the reaction.

Freeze-dried mixtures of the two substances (1 $\frac{1}{2}$ moles glucose/amino group, $p \mathrm{H}$ on re-solution $6 \cdot 5$ ) were shown to react with progressively increasing complexity as the relative humidity increased, until at about 60 per cent relative humidity all the changes characteristic of the Maillard reaction in true solution ${ }^{2}$ were occurring. The reaction products were examined by descending paper chromatography with 80 per cent aqueous propanol as solvent.

At 20 per cent relative humidity a simple one-toone glucose-amino combination took place and gave rise to a spot at $R_{F} 0.1(A)$ which had all the properties described by Gottschalk and Partridge ${ }^{3}$. Glucose and $\alpha-N$-acetyl-L-lysine were clearly separated at $R_{F} 0 \cdot 3$ and $0 \cdot 2$. At 30 per cent relative humidity there appeared, in a characteristic band on the rear edge of the first spot, a second compound $(B)$ which gave a bright purple fluorescence under an ultra-violet lamp and on elution showed a pronounced absorption peak at $2880 \mathrm{~A}$. Reduction of ammoniacal silver nitrate and ferricyanide at $p \mathrm{H} 5$ occurred in the area $A B$. At 40 per cent relative humidity a series of non-reducing pale-blue-fluorescent compounds $(C)$ appeared at $R_{F}$ 's $0.05,0.03$ and 0.01 , while brown products $(D)$, which had now begun to accompany the reaction, remained stationary. Compounds $C$ and $D$ could be reproduced by storing the isolated amino-glucose complex $A$ at 40 per cent relative humidity, and this, combined with a progressive movement towards the visible region of a poorly defined peak in their absorption spectra (reaching $3280 \mathrm{~A}$. in the brown compounds), suggests that they are successive degradation products of the complex.

At 60 per cent relative humidity the pattern of the fluorescent compounds, though similar, was more complex and has not yet been examined in detail. It is interesting to note, however, that several of the complex effects, including browning, fall in $p \mathrm{H}$ and loss of glucose in excess of the one-to-one relationship, reached a maximum at this humidity, whereas the maximum rate of loss of amino groups occurs at 40 per cent relative humidity. A similar behaviour has already been observed in the protein-sugar reaction 4 .

The precise chemical nature of compounds $A$ and $B$ still remains to be determined. Both appear to be primary products of a one-to-one reaction, but neither liberates glucose on acid hydrolysis nor undergoes reduction by hydrogen and palladium black at room temperature and pressure. There is therefore as yet no direct evidence for an N-glycoside or isoglucosamine structure of the type well known in the aromatic series. Compound $A$ appears to be one of the main intermediaries in the browning sequence; but sufficient browning to account for that of the intact systems could only be achieved by storage of $A$ with all the slower-moving compounds (except $D$ ). This mixture browned two or three times as rapidly as $A$ alone. At least one of these other compounds is therefore also involved, possibly in a catalytic role, since only $A$ browns when a developed paper chromatogram is heated.

These experiments, and others with a preparation of polylysine of molecular weight $10-15,000$, were carried out with the object of assessing the extent of the contribution of the terminal amino groups of 